\title{
Improving Profitability of the Network Distribution by protecting Power Transformers with a Current Limiting Technology Device
}

\author{
T. MADIBA, M. SITI and A.A JIMOH
}

\author{
Department of Electrical Engineering \\ Tshwane University of Technology \\ Campus of Pretoria - Gauteng Province, 0001 South Africa \\ Phone number: +27 748142311, e-mail: sinfocimb@yahoo.fr, willysiti@yahoo.com, jimohAA@tut.ac.za.
}

\begin{abstract}
This paper presents the improvement of the profitability of the Network Distribution, when power transformers are protected only with Fault Current Limiting Technologies (FCLT). In assessing the total benefits of the Network Distributions from normal to post-fault conditions, we will compare two kinds of profitability taking in account the impact of Circuit Breakers (CBs) and FCL used separately as protection devices during the life span of power transformers. This study will be performed using MATLAB/SIMULINK as software and it was concluded that the FCL perfectly improves the profitability of the Network Distribution.
\end{abstract}

\section{Key words}

MATLAB/SIMULINK, FCL, CBs, Total benefits, Profitability.

\section{Introduction}

In distribution network, one of the major targets is to protect the equipments, as well as the consumers. Under normal condition, the protection devices used in the circuit to protect the different equipments should not have a big disadvantage such as power loss, the voltage drop into the network distribution. The need of customer's power quality is very important with the aim to protect their expensive equipments. During the normal conditions in the power system, a fault current limiter as well as CBs should not appear totally in the circuit because of the voltage drop and unforeseen breaking action. Therefore, during the fault condition of the network distribution, there is power loss and when the protection device comes to secure the expensive equipments in the circuit, the level of current under post fault condition voltage obtained at the primary side should not be so high for limiting the power loss under this condition. [1], [2], [3], [4], [5].

In this paper, we have studied the comparison between two types of profitability for two network distributions of which one includes $\mathrm{CBs}$ as protection device of power transformers and another one includes FCL for accomplishing the same action. By evaluating the Total Benefits Costs during the normal and fault operation, also we have evaluated the lifecycle cost after the lifetime of power transformer with conventional copper. The results are presented using MATLAB/SIMULINK.

\section{CBs and FCL in two different Networks Distribution as protection devices}

We considered two types of Network Distributions containing each the source, the line, two power transformers to be protected and the load characterizing the utility. In Figures 1 and 2 below are presented these Networks just stated above with theirs components respectively. As said above, the first Network uses the $\mathrm{CBs}$ as protection device for breaking the three phase system under fault operation and the second uses the FCL for limiting the fault current under abnormal operation of the three phase system.

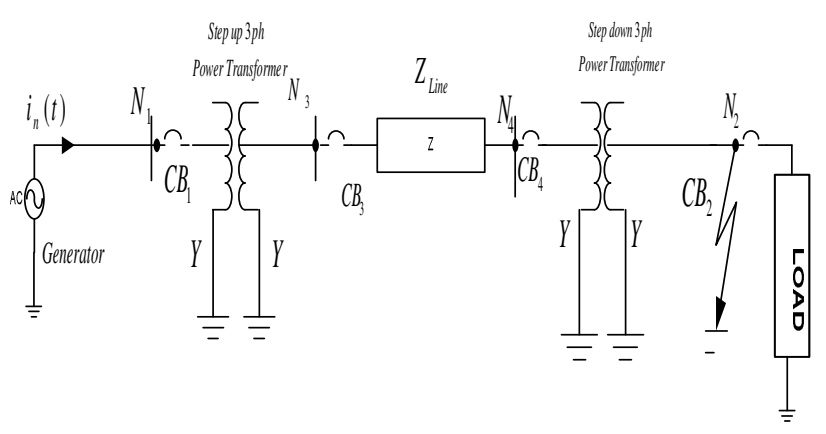

Figure 1: Network Distribution with CBs

The FCL used for limiting the short circuit current under fault condition is a Thyristor Controlled Series Reactor (TCSR) which is presented in Figure 3 below. The current under normal operation is expressed below by the equation 1 :

$i_{a b c}(t)=I_{\max } \times\left[\sin \left(\omega t+\theta_{a b c}+\eta-\phi_{a b c}\right)\right]$

In which

$\theta_{a b c}$ is the source voltage angle for each phase $\eta$ is the firing angle of the Thyristors

$\phi_{a b c}$ is the total impedance angle under normal condition for each phase 


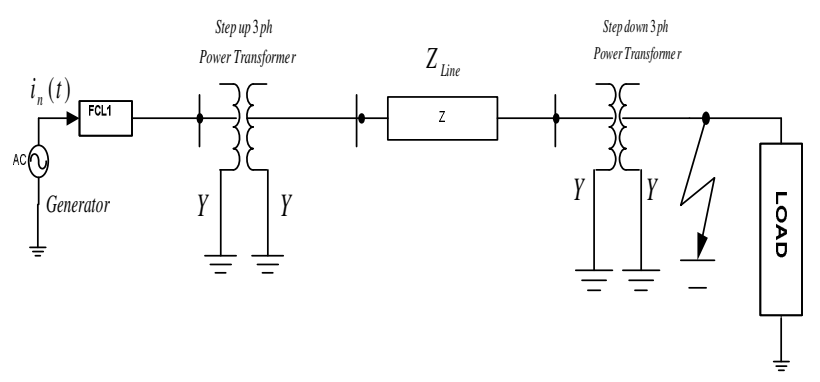

Figure 2: Network Distribution with FCL

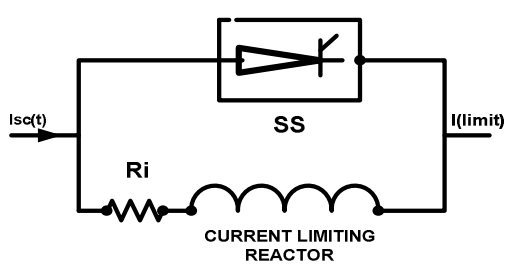

Figure 3: Model of FCL-TCSR

For both cases, the fault considered is the $3 \mathrm{LG}$ fault condition. For this $3 \mathrm{LG}$ fault, the currents and total impedance under short circuit are respectively given by the equations (2) and (3) below:

$$
\begin{gathered}
I_{a g}^{\prime}=m I_{a}\left\{\left[\sin \left(\omega t+\theta_{a}-\phi_{a b c g}\right)\right]+\left[\sin \left(\theta_{a}-\phi_{a b c g}\right)\right] e^{-\alpha_{2} t}\right\} \\
I_{b g}^{\prime}=m I_{b}\left\{\left[\sin \left(\omega t+\theta_{b}-\phi_{a b c g}\right)\right]+\left[\sin \left(\theta_{b}-\phi_{a b c g}\right)\right] e^{-\beta_{2} t}\right\} \\
I_{c g}^{\prime}=m I_{c}\left\{\left[\sin \left(\omega t+\theta_{c}-\phi_{a b c g}\right)\right]+\left[\sin \left(\theta_{c}-\phi_{a b c g}\right)\right] e^{-\xi_{2} t}\right\} \\
Z_{a b c g}=Z_{g}+\sum_{i=1}^{9} Z_{i a b c}+k^{2}\left[\sum_{i=1}^{6} R_{C B}+\sum_{j=1}^{6} Z_{j a b c}\right] \\
Z_{a b c g}=\left|Z_{a b c}\right| \angle \phi_{a b c g}
\end{gathered}
$$

Where

$\alpha, \beta, \xi$ represent the ratio between resistances and inductances under short-circuit.

$Z_{i(a b c)}$ are all impedances from primary side of power transformers.

$Z_{j(a b c)}$ all impedances from secondary side referred to primary side.

\section{Post-fault Condition}

This part is defined by the actions realized with the previous protection equipments in the circuit under abnormal operation. We are going to express the equations below of this condition related with the impact of CBs as well as of FCL in the circuit.

Applying CBs in the circuit, we have as stated above, the equations (4) and (5):

$$
\begin{aligned}
& I_{a g}^{\prime}=I_{b g}^{\prime}=I_{c g}^{\prime}=K(10 \text { Cycles }) \times I_{\lim } \\
& I_{\lim }=I_{a g}^{\prime} \times\left[1+2 e^{[-4 \pi \tau /(X / R)]}\right]^{-0.5} \\
& \Delta P_{1}=3\left[K(\tau) \times I^{\prime}{ }_{a g}\right] \times\left[\sum_{i=1}^{n} R_{i}\right] \\
& \tau=0.5 \text { cycles }
\end{aligned}
$$

$\mathrm{I}_{\mathrm{lim}}$ is the magnitude of acceptable current.

$I_{a g}$ is the magnitude of fault current

Applying FCL in the circuit, we have equations (6), (7), and (8) below:

$$
\begin{aligned}
& I_{\lim }=p I_{a}\left\{\left[\sin \left(\omega t+\theta_{a}-\phi_{a b c g}\right)\right]+\left[\sin \left(\theta_{a}-\phi_{a b c g}\right)\right] e^{-\alpha_{2} t}\right\} \\
& I_{\lim }=p I_{b}\left\{\left[\sin \left(\omega t+\theta_{b}-\phi_{a b c g}\right)\right]+\left[\sin \left(\theta_{b}-\phi_{a b c g}\right)\right] e^{-\beta_{2} t}\right\} \\
& I_{\lim }=p I_{c}\left\{\left[\sin \left(\omega t+\theta_{c}-\phi_{a b c g}\right)\right]+\left[\sin \left(\theta_{c}-\phi_{a b c g}\right)\right] e^{-\xi_{2} t}\right\} \\
& L_{F C L}=\frac{V_{F C L} \times \Delta t}{I_{a} \times(m-p)} \\
& \Delta P_{2}=3\left[I_{n}(p-1)\right]^{2} \times\left[\sum_{i=1}^{n} R_{i}\right]
\end{aligned}
$$

Where

$p$ is the number of times the rated current $\mathrm{V}_{\mathrm{FCL}}$ the voltage across the FCL

$$
\Delta t \text {. }
$$
is the short circuit time

\section{Profitability of the Network Distribution}

This step of study is characterized by two major parts especially the normal and the fault time of the Network Distribution. For our clearance, we will evaluate both the power loss by both two types of protection devices CBs and FCL separately which will define the Life span of the power transformers.

\section{A. Profit with the use of $C B s$}

The Total Capital invested of the Network Distribution is defined in this case by the equation (9) below [6], [9], [10], [11]:

$$
C_{i}=\sum_{i=1}^{n} C_{e q}=C_{G}+C_{T}+C_{C B s}+C_{\text {line }}+C_{\text {Mce }}(9),
$$

Where

$\mathrm{C}_{\mathrm{i}}$ is the capital invest of all equipments

$\mathrm{C}_{\mathrm{G}}$ is the cost of the Generator

$\mathrm{C}_{\mathrm{T}}$ is the cost of power transformers to protect

$\mathrm{C}_{\text {line }}$ is the cost of the power cable of connection

$\mathrm{C}_{\text {Mce }}$ is the cost of replacements and maintenance

$\mathrm{C}_{\text {prof }}$ is the Profitability of the Network after $n$ years

The ratio between the times of fault condition and of normal condition is 0.1 and it can be expressed in term of annual time. Taking in account the cost of electricity and the lifetime of three phase power transformers, the equation (10) defines the Lifecycle Cost for this case.

$$
T_{c c_{\text {loss } 1}}=\Delta E_{1} \times C_{K W} \times \frac{(1+r)^{n}-1}{r \times(1+r)^{n}}
$$

$T_{c c_{\text {loss }}}=$ the total amount of Power Dissipation expressed in dollars $(\$)$,

From the equation (10), we can deduce the annual energy in term of time.

$\Delta E_{1}=\Delta P_{1} * 876$ is the value of Power loss after one year expressed in $\mathrm{KWhr}$. 
$\mathrm{C}$ equal to the cost per $\mathrm{KWh}$ considered actually at $C_{K W} \cong 0,05 \$ / K W h$ The capital cost for the lifecycle cost, generally $r=3 \%[7,8]$, and $\mathrm{n}$ is the number of years, or the lifetime of power transformers which generally considered equal to 25 years [12], [13].

\section{B. Profit with the use of FCL}

Applying the FCL in the circuit as protection device of power transformer, the Total Capital invests of the Network Distribution is defined in this case by the equation below (11):

$C_{i}=\sum_{i=1}^{n} C_{e q}=C_{G}+C_{T}+C_{F C L}+C_{\text {line }}+C_{\text {Mce }}(11)$,

Where

$\mathrm{C}_{\mathrm{FCL}}$ is the cost invest of Fault Current Limiter FCL

Referring to the equation (10) above, the equation (12) below represents the LCC of the power loss related with application of Fault Current Limiter in the circuit under fault condition

$$
T_{c c_{\text {loss } 2}}=\Delta E_{2} \times C_{K W} \times \frac{(1+r)^{n}-1}{r \times(1+r)^{n}}
$$

The total actualised benefits of the Network Distribution during the life span of power transformer to protect is expressed as the difference between the actualised Capital investment and Benefits after 25 years considered as lifetime of power transformers. Related with what told above, the equations (13), (14) and (15) define the Profitability of the Network Distribution.

$C_{i, a c t}=C_{i} \times(1+r)^{n}$

$B_{a c t}=P_{K W} \times 8760 \times C_{K W} \times\left[\sum_{n=1}^{n=25}(1+r)^{n}\right]$

$C_{\text {Prof }}=B_{a c t}-C_{i, a c t}$

Where

$\mathrm{C}_{\mathrm{i} \text {, act }}$ is the actualised capital investment after $\mathrm{n}$ years

$\mathrm{B}_{\mathrm{act}}$ is the actualised benefits after $\mathrm{n}$ years

\section{Results of the Study}

For getting the confirmation of this study, we simulated a system constituted by the source, transmission lines, two three phase power transformers to protect, and a balanced load.

\section{A. Data}

Two three phase power transformers (Yyn):

$$
\begin{aligned}
& 250 M V A, 18 / 300 K V, \% Z_{e q(T 1)}=5 \\
& 250 M V A, 300 / 132 K V, \% Z_{e q(T 2)}=14.62 \\
& C_{T}=149,368,842 \$
\end{aligned}
$$

Source characteristics per phase:

$$
\begin{aligned}
& V=10.3923 K V, H=3 s, X_{d}=1.7199, X_{d^{\prime}}=0.23 \\
& T_{d o^{\prime \prime}}=0.029, X_{q}=1.6598, X_{q^{\prime \prime}}=0.29, T_{q o^{\prime \prime}}=0.034 \\
& P=55 M W, Q=9.031 M V A R, C_{G}=275 \times 10^{5} \$
\end{aligned}
$$

Transmission lines primary and secondary sides for each phase:

$L_{L}=3 \mathrm{mH} / \mathrm{km}, R_{L}=0.15 \Omega / \mathrm{km}$, Lengh $\cong 80 \mathrm{Km}$

$C_{\text {line }}=8 \times 10^{5} \$$

AC Controller and Circuit Breakers: same value $R_{\text {on }}=0.001 \Omega, \eta=90^{\circ}, m=34, p=2$

$C_{C B s}=2 \times 10^{5} \$, C_{F C L}=25,000 \$$

Cost of maintenance and yearly exploitation

$$
C_{a c t, M c e}=C_{M c e} \times\left[\sum_{n=1}^{25}(1+r)^{n}\right]
$$

The balanced Load characterized by: $P=60 M W, Q=20 M V A R, f=50 H z$

\section{B. Explanation of Results}

Applying from equations 1 to 8, the current's magnitude of normal, fault and post-fault conditions and are expressed below:

Node $1: I_{a b c}=2.5 \mathrm{KA}$, Node $2: I_{a b c}=0.36 \mathrm{KA}$

Node $3 \cong$ Node $4: I_{a b c}=0.151 K A$

Node $1: I_{a b c}=85 \mathrm{KA}$, Node $2: I_{a b c}=12.2 \mathrm{KA}$

Node $3 \cong$ Node $4: I_{a b c}=5.2 \mathrm{KA}$

Post-fault current with FCL after $1 / 2$ cycles

Node $1: I_{a b c}=5 \mathrm{KA}$, Node $2: I_{a b c}=0.72 \mathrm{KA}$

Node $3 \cong$ Node $4: I_{a b c}=0.302 \mathrm{KA}, L_{F C L}=0.05 \mathrm{H}$

Post-fault Currents with CBs after $1 / 2$ cycles

Node $1: I_{a b c}=60.5 \mathrm{KA}$, Node $2: I_{a b c}=8.68 \mathrm{KA}$

Node $3 \cong$ Node $4: I_{a b c}=3.7 \mathrm{KA}$

Applying equations 10 and 11, the lifecycle cost related with the CBs and FCL in the Network Distribution is defined by the table 1 below and presented in Figure 4, 5 and 6.

Table1. Lifecycle Cost (CBs \& FCL)

\begin{tabular}{|c|c|c|}
\hline$n($ years $)$ & $T_{c c}\left[10^{6} \$\right] \mathbf{C B s}$ & $T_{c c}\left[10^{6} \$\right] F C L$ \\
\hline 2 & 1835.53 & 31.96 \\
\hline 5 & 4392.22 & 76.49 \\
\hline 8 & 6732.2 & 117.234 \\
\hline 10 & 8180.27 & 142.45 \\
\hline 12 & 9542.05 & 166.17 \\
\hline 17 & 12630.03 & 219.94 \\
\hline 20 & 14269.92 & 248.498 \\
\hline 25 & 16699.1 & 290.8 \\
\hline
\end{tabular}


Applying both costs and equations 13, 14 and 15, we have the results as follow in the table 2 below which are presented in Figure 7, 8 and 9.

Table 2. Profitability of Network Distribution with the use of

\begin{tabular}{|c|c|c|c|c|}
\multicolumn{7}{|c|}{ CB, FCL } \\
\hline years & $\begin{array}{c}C_{i a}, C B \\
{\left[10^{8} \$\right]}\end{array}$ & $\begin{array}{c}C_{i a}, F C L \\
{\left[10^{8} \$\right]}\end{array}$ & $\begin{array}{c}\operatorname{Pr} f_{, C B} \\
{\left[10^{8} \$\right]}\end{array}$ & $\begin{array}{l}\operatorname{Pr}{ }_{, F C L} \\
{\left[10^{8} \$\right]}\end{array}$ \\
\hline 1 & 1.9 & 1.886 & -1.617 & -1.616 \\
\hline 2 & 1.94 & 1.89 & -1.395 & -1.341 \\
\hline 3 & 2.002 & 2.001 & -1.166 & 1.165 \\
\hline 4 & 2.1 & 2.06 & -0.930 & -0.929 \\
\hline 5 & 2.12 & 2.12 & -0.687 & -0.687 \\
\hline 6 & 2.19 & 2.18 & -0.437 & -0.436 \\
\hline 7 & 2.3 & 2.25 & -0.180 & -0.179 \\
\hline 8 & 2.32 & 2.3 & 0.084 & 0.086 \\
\hline 12 & 2.613 & 2.611 & 0.885 & 0.821 \\
\hline 17 & 3.03 & 3.02 & 2.517 & 2.519 \\
\hline 20 & 3.31 & 3.308 & 5.873 & 5.875 \\
\hline 25 & 3.84 & 3.335 & 7.942 & 8.441 \\
\hline
\end{tabular}

For the same fault condition, the Figure 10 gives the variability of active power. When FCL applied we have Figure 11 and when CBs applied in the circuit under fault we have Figure 12 which shows that the power under post fault condition is higher.

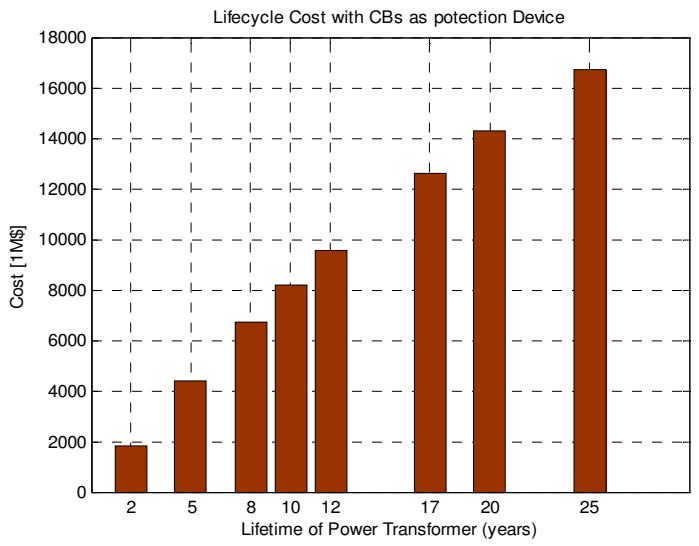

Figure 4: Lifecycle Cost with CB in use

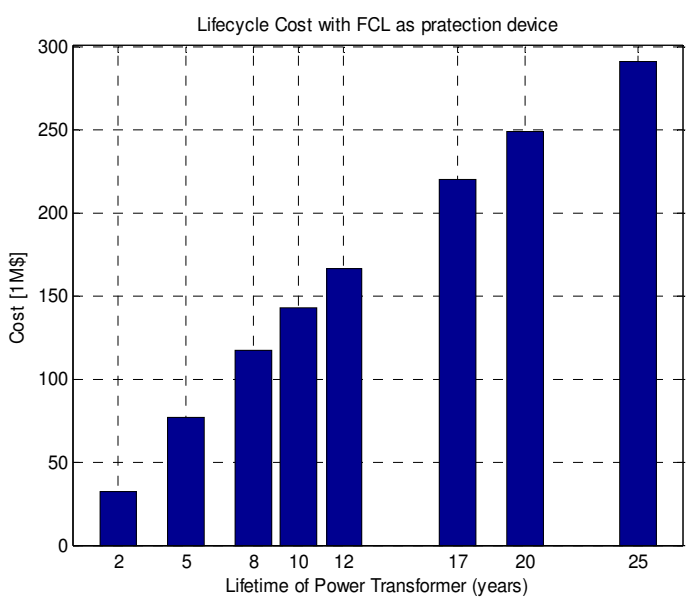

Figure 5: Lifecycle Cost with FCL in use

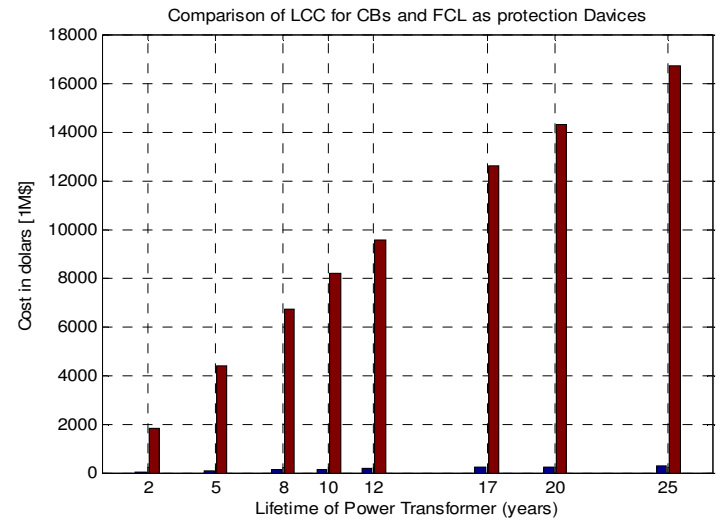

Figure 6: Comparison of LCC of CBs and FCL

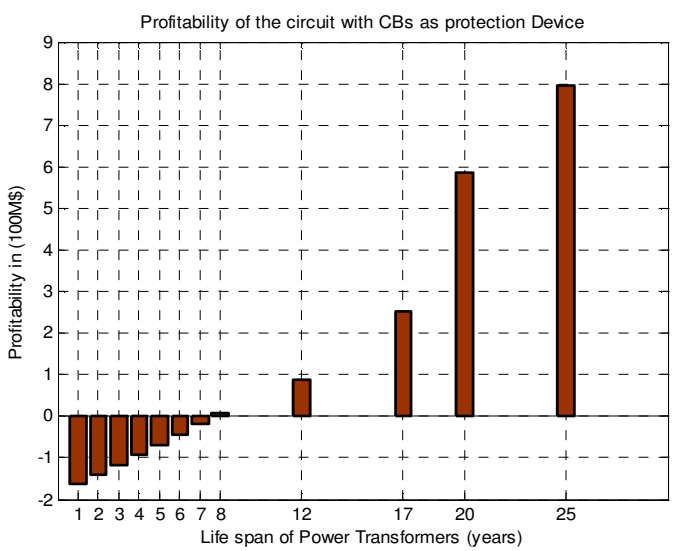

Figure 7: Profit of Network with use of CBs Profitability with $\mathrm{FCL}$ as protection Device

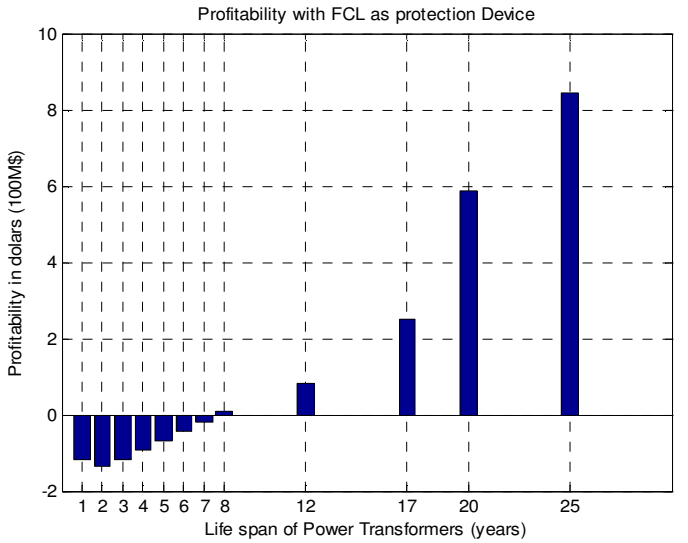

Figure 8: Profit of Network with use of FCL

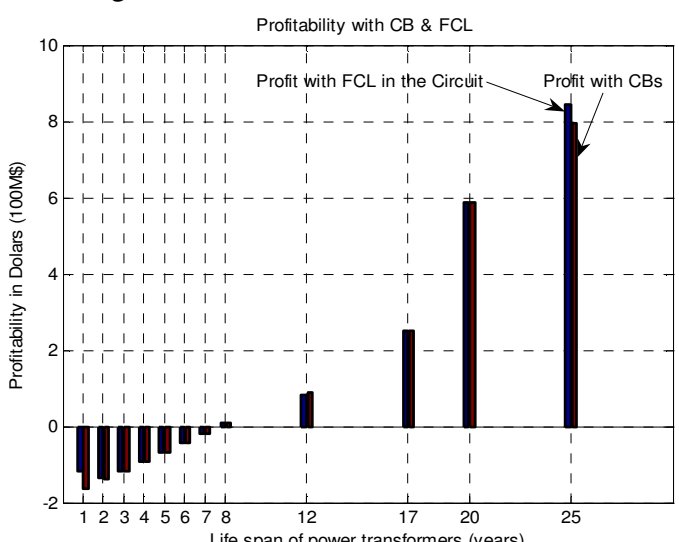

Figure 9: Comparison of CBs and FCL's Profits 


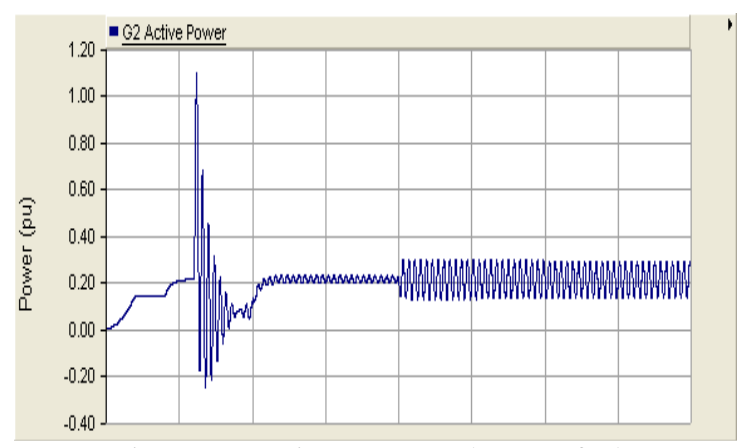

Figure 10: Active Power under 3LG fault

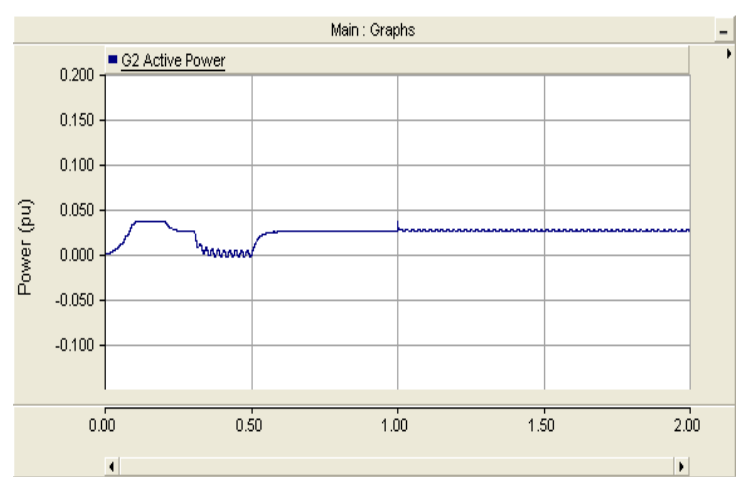

Figure 11: Active Power with protection of FCL

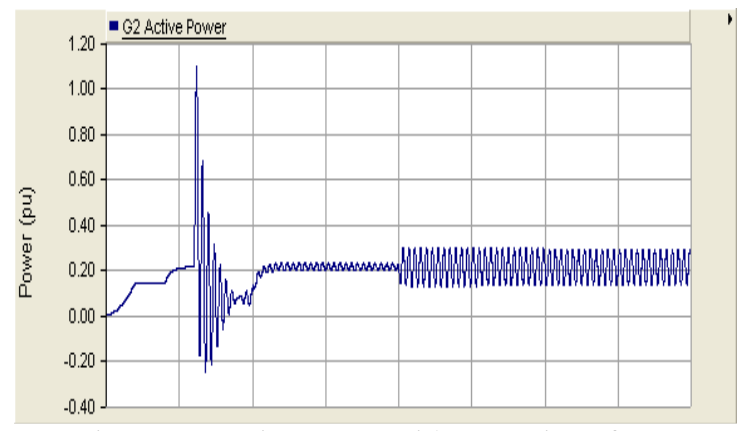

Figure 12: Active Power with protection of CB

\section{Conclusion}

During this study, we observed that there is a difference between protection devices such as CBs and FCL. About the Lifecycle Cost, a Network Distribution with CBs in use will get a high LCC because of the CB's Cost of replacement, their short lifetime and the high power loss caused by their presence in the circuit. Therefore, one FCL has a less Cost and it can be used where four CBs are needed during all the life span of power transformers. The comparison related with the Profitability, a Network Distribution with FCL in use will get a high profit with the same active power required to be delivered to customer side. For companies in charge of Electrical Distribution, the use of FCL as protection devices is very perfect because of the less LCC and high profit.

\section{Acknowledgment}

The authors acknowledge the financial supports of Eskom through the Tertiary Education Support Program and the National Research Foundation's IRDP program for this work.

\section{References}

[1] Alstom,"Air Core Reactor", www.tde.alstom.com

[2] ESTU/DTI,'Likely changes to Network Design as a result of significant embedded Generation", A Technology Ltd, 2001.

[3] ABB,"HTS fault current limiter", www.abb.com

[4] Siemens," Superconductive Current Limiter", www.siemens.com

[5] TEPCO,’Development of fault current limiters", http:// www.tepco.co.jp/rd/power/dtyodend/fcl/fcl-e.html

[6] N. Magnusson, G. Mugala, N. Schonborg, and Hornfeldt,"Critical Currents in AC External Magnetic Field", IEEE Trans. Appl. Supercond., 9, p. 785, 1999.

[7] N. Magnusson, G. Mugala, N. Schonborg, and Hornfeldt,"Temperature dependence of AC losses in HighTemperature Superconductors", Inst. Phys. Conf. Ser., 167, p. 891, 2000.

[8] Passi J., et al,"Superconducting Power Link for Power transmission and fault current limitation", Physica, C354 (2001), pp1-4.

[9] Massachusetts Institute of Technology 22.68J/2.64J,"Superconducting Magnet", February 6, 2003.

[10] Ronnie B., Jan D., Hans D.K., Katsuaki F., Mayur K., Manuel M., Mike McD., Ivo P., "The Potential for Global Energy Savings from High Efficiency Distribution Transformers", pp23-26, February 2005.

[11] B.S. Blanchard, W.J. Fabrycky. Systems engineering and analysis. Prentice Hall, New Jersey, USA, 1998

[12] ISO 14040 - Environmental management - life cycle assessment: principles and framework, Berlin, Beuth, 1997.

[13] IKP University of Stuttgart and PE Europe GmbH. "GaBi 4: the software for environmental process and product optimization", 2006. Available: http://www.gabisoftware.com/index.html. 